4.1 in chromaffin cells, and immunofluorescence and immunoperoxidase staining with affinity-purified anti-red cell 4.1 shows that the chromaffin cell-related protein is concentrated in the cell periphery, associated either with the plasma membrane or with the cortical cytoskeleton. Therefore, the chromaffin cell form of protein 4.1 could be a target for protein kinases in the regulation of the peripheral cytoskeleton of the chromaffin cell, in particular of the association of cortical actin filaments with fodrin and the plasma membrane.

We agree with Siegel's suggestion that there are likely to be common molecular mechanisms in the control of exocytosis in chromaffin cells, nerve terminals and also other cells. In the case of the regulation of the interaction of actin filaments with fodrin and membranes it seems that similar control mechanisms may occur, but with different members of the same functional family of molecules as targets in different cell types.

Robert D. Burgoyne MRC Secretary Control Group,

The Physiological Laboratory,

University of Liverpool,

PO Box 147,

Liverpool L69 3BX, UK

Biological Laboratory,

ANTHONY J. BAINES

University of Kent,

Canterbury,

Kent CT2 8NJ, UK

1. Burgoyne, R.D. \& Cheek, T.R. Nature 326, 448 (1987).

Baines. A J Nature 326.646(1987)

Siegel. D Nature 327, 467 (1987)

. Bahler. M. \& Greengard, P. Nature 326, 704-707 (1987).

Burgoyne R.D. \& Geisow. M.J. FEBS Lett. 131, 127-131 (1981).

6. Fried G. et al. Proc. natn. Acad. Sci. U.S.A. 79, 2717-2721 (1982)

Navone. F. et al. J. Cell Biol. 103, 2511-2527 (1986)

8. Baines. A.J. \& Bennett, V. Nature 315. 410-413 (1985)

\section{Artificial guide stars for adaptive imaging}

SiR-The experiments of Thompson and Gardner ${ }^{1}$ followed proposals by Foy and Labeyrie $^{2}$ to create artificial 'guide stars' for adaptive imaging in astronomy by the backscattering from the upper atmosphere of laser pulses projected from a telescope. It has been suggested that if sufficient energy could be returned from a thin scattering layer it could provide the information required to drive adaptive optical systems able to compensate for the atmospheric degradation of images of faint objects of astronomical interest.

There appear to be difficulties with this proposal, as in perhaps the simplest possible case the backscattered light provides no information at all about refraction in the lower atmosphere.

Suppose, for example, that the scattering layer is very distant and that the aperture of the telescope/projector may be completely covered by a thin transparent wedge whose only effect is to refract light passing through it through a small angle. The image of a distant real object will move if this wedge is introduced, but an image formed by backscattering will be unaffected because the incoming beam will precisely retrace its outgoing path.

Complexities introduced by random variations of refraction across single or multiple apertures do not seem likely to improve this situation, and it therefore seems improbable that techniques of this kind will yield the benefits that have been suggested.

Royal Greenwich Observatory,

Hailsham,

East Sussex BN27 1RP, UK

1. Thompson, L.A. \& Gardner, C.S. Nature 328, 229-231 (1987).

. Foy, R. \& Labeyrie, A. Astr. Astrophys. 152, L29-L31 (1985).

ThOMPSON AND GARDNER REPLY-There are three potential problems that might cause a laser guide star to move relative to natural background stars: laser jitter, non-sidereal motion of the telescope, and low frequency atmospheric disturbances.

Problems of laser jitter can be removed by monitoring the laser beam as it leaves the telescope, and any detected motion can be nulled in the computer which analyses the wave front error. Telescope jitter can be removed by locking the instrument onto bright natural stars; these stars need not be in the isoplanatic patch.

The problem of low frequency atmospheric disturbances is more subtle, but it too can be corrected. If the natural guide stars used to compensate for telescope jitter are spaced over a large area in the sky $\left(5^{\circ}\right.$ to $10^{\circ}$ patch $)$, the average low frequency tilt will be zero. Then if we simultaneously monitor the motion of a natural star near the laser target, its motion will show evidence of all low frequency disturbances occurring at the target. This signal can also be sent to the computer which analyses the wave front error, and the computer can make the required corrections.

Even if the multiple guide star technique fails to remove all low frequency wave front errors, there is a less acceptable but safe fall-back solution. A moderately fast data recording system can be used at the final focal plane, and the adaptively sharpened images can' be recorded with relatively short exposure times. These short exposures can be recentred and coadded ex post facto, thereby giving a sharpened and stable image.

LAIRD THOMPSON Chet Gardner

Department of Astronomy,

University of Illinois at

Urbana-Champaign,

1011 West Springfield Avenue,

Urbana, Illinois 61801, USA

\section{Red Queen versus Tangled Bank models}

SIR-Burt and Bell ${ }^{1}$ present an analysis of chiasma frequency in mammals, which they claim supports the theory that genetic recombination is maintained by selection pressures resulting from host-pathogen interactions, which cause cyclical fluctuations in the nature of selectively favoured gene combinations (the Red Queen hypothesis ${ }^{2}$ ). The basis for this claim is that the excess of chiasma frequency per individual over haploid chromosome number is positively correlated with age to maturity (which they equate with generation time); they assert that this correlation is predicted by the Red Queen model, but not by the only other model that they consider to be a viable alternative - the model of sib-competition in a spatially varying environment ${ }^{3,4}$, which they term the Tangled Bank model.

Their reasoning is that the timescale for interactions between mammals and their pathogens is such that species with a long generation time will be more likely to encounter a change in selection pressure over a small number of generations, compared to species with short generations, and hence will be more likely to evolve high rates of recombination. They also claim that species with large litter size will be more likely to experience sib-competition; because these tend to have short generations, the Tangled Bank model predicts a negative correlation with generation time.

There are several difficulties with this study. In the first place, the authors' treat each species as an independent data point in their correlation tests. It is a wellknown principle of the comparative method that such correlations are biased in the direction of spuriously positive results if there is a lack of evolutionary independence between groups of data points ${ }^{5}$. Inspection of Burt and Bell's Fig. 1 shows that there are many points contributed by species of primates and rodents; much of their correlation is evidently due to the fact that rodents tend to have low chiasma frequencies per bivalent and primates high ones. It is not at all clear what this means, in view of the many biological differences between these groups. It could, for example, be the case that sib-competition is more intense in the highly social primates, leading to a greater advantage to high recombination through the Tangled Bank model. Such sibcompetition has been invoked as a cause of female-biased sex ratios in primates ${ }^{6}$.

Second, their rejection of all possible explanations except the Red Queen and Tangled Bank models, on the basis of Bell's survey ${ }^{2}$ of the comparative data on the taxonomic distribution of asexuality in animals, is not accepted by many workers in the field. As pointed out in at least 